\title{
CARACTERISTICAS DE LAS INTELIGENCIAS MÚLTIPLES DE LOS ALUMNOS DEL SEXTO GRADO DE PRIMARIA DE LA INSTITUCIÓN EDUCATIVA CRISTO REY - AÑO 2006
}

Responsable : Mgr. Humberto Vargas Pichón Miembros : Mgr. Segundo Manuel Alvarado Contreras

\section{RESUMEN}

El presente trabajo de Investigación es un estudio no experimental de tipo correlacional, de corte transversal, que se efectuó durante el periodo abril agosto 2007 en la Institución Educativa "Cristo Rey" de Tacna.

Mediante el objetivo general se pretendió "Establecer una correlación entre el nivel de desarrollo de las inteligencias múltiples y el Rendimiento Académico de los Alumnos de primero y segundo grado de secundaria de la Institución Educativa Cristo Rey de Tacna" en respuesta a la interrogante ¿En qué medida existe una correlación directa y significtiva entre el nivel de desarrollo de las inteligencias múltiples y el Rendimiento Académico de los alumnos de primero y segundo grado de secundaria de la Institución Educativa "Cristo Rey" de Tacna? Sosteniendo la hipótesis de que: "Existe una correlación directa y significativa entre el nivel de desarrollo de las inteligencias múltiples y el Rendimiento Académico de los alumnos de primero y segundo grado de secundaria de la Institución Educativa "Cristo Rey"de Tacna.

El diseño muestral es probabilístico sistemático. Y considerando el muestreo estratificado se trabajó con una muestra de 44 alumnos de primero y segundo grado de secundaria de la Institución Educativa "Cristo Rey" de Tacna.

Se utilizó el Programa Estadistico SPSS V-12-e y el Microsoft Excel XP para el procesamiento de datos y la elaboración de los cuadros y gráficos estadisticos asi como la prueba de hipótesis correspondiente, lo que nos permitió obtener la conclusión principal correspondiente.
ABSTRACT

The present Work of Investigation is a nonExperimental study of corelational type, of cross section, that took place during the period April to August 2007 in the Educative Institution "Christ King" of Tacna.

By means of the general mission it was tried "To establish a correlation between the level of development of Multiple Intelligences and the Academic Yield of the Students of first and second secondary degree of of the Educative Institution Christ King of Tacna" in answer to the question To what extent exists a direct and significtiva correlation between the level of development of multiple intelligences and the Academic Yield of the students of first and second secondary degree of of the Educative Institution Christ King of Tacna. Maintaining the hypothesis that: "a direct and significant correlation between the level of development of Multiple Intelligences and the Academic Yield of the students of first and second secondary degree of of the Educative Institution Christ Exists King of Tacna".

The design sample is probabilistico systematic and considering the sampling stratified it worked with a sample of 44 students of first and second secondary degree of of the Educative Institution "Christ King" of Tacna.

It was used Statistical Program SPSS V-12-e and the Microsoft Excel XP for the data processing and the statistical elaboration of the pictures and graphs as well as the test of corresponding hypothesis, which allowed us to obtain the corresponding main conclusion.

\section{INTRODUCCIÓN}

En un pasado, no muy lejano, habia un cierto acuerdo sobre el concepto de inteligencia y se pensaba que todas las personas aprendian casi de la misma manera y, por lo tanto, existía sólo una forma de enseñar.

Hoy, sin embargo, se advierte la existencia de diferentes 'estilos cognitivos', lo que implica que los métodos de enseñanza se adapten a estos estilos particulares del o de los que aprenden.

Por otro lado, una de las dimensiones más importantes en el proceso de enseñanza aprendizaje - lo constituye el Rendimiento Académico del alumno.
Cuando se trata de evaluar el Rendimiento Académico y cómo mejorarlo, se analizan en mayor o menor grado los factores que pueden influir en él; generalmente se consideran, entre otros, factores socioeconómicos, la amplitud de los programas de estudio, las metodologías de enseñanza utilizadas, la dificultad de emplear una enseñanza personalizada, los conceptos previos que tienen los alumnos, asi como el nivel de pensamiento formal de los mismos.

Debido a todo esto es que en la presente investigación se demostró, con un nivel de significancia de 0,10 , que "Existe una correlación directa y significativa entre el nivel de desarrollo de las Inteligencias múltiples y el Rendimiento Académico de los alumnos de primero y segundo grado de secundaria de la Institución Educativa "Cristo Rey" de Tacna" 


\section{1) Desarrollo del Tema}

\begin{tabular}{|c|c|}
\hline Inteligencia & Componentes centrales \\
\hline Lingüística & $\begin{array}{l}\text { Sensibilidad a los sonidos, la } \\
\text { estructura, los significados y las } \\
\text { funciones de las palabras y el } \\
\text { lenguaje. }\end{array}$ \\
\hline $\begin{array}{l}\text { Lógico- } \\
\text { matemática }\end{array}$ & $\begin{array}{l}\text { Sensibilidad y capacidad para } \\
\text { discernir los esquemas numérico o } \\
\text { lógicos; la habilidad para manejar } \\
\text { cadenas de razonamientos largas. }\end{array}$ \\
\hline Espacial & $\begin{array}{l}\text { Capacidad para percibir con } \\
\text { precisión el mundo visual y espacial, } \\
\text { y la habilidad para efectuar } \\
\text { transformaciones en las } \\
\text { percepciones iniciales que se hayan } \\
\text { tenido. }\end{array}$ \\
\hline $\begin{array}{l}\text { Corporal- } \\
\text { Kinética }\end{array}$ & $\begin{array}{l}\text { Habilidad para controlar los } \\
\text { movimientos del cuerpo y manejar } \\
\text { objetos con destreza. }\end{array}$ \\
\hline Interpersonal & $\begin{array}{l}\text { Capacidad para discernir y responder } \\
\text { de manera adecuada a los estados } \\
\text { de ánimo, los temperamentos, las } \\
\text { motivaciones y los deseos de otras } \\
\text { personas. }\end{array}$ \\
\hline Intrapersonal & $\begin{array}{l}\text { Acceso a los sentimientos propios y } \\
\text { habilidad para discernir las } \\
\text { emociones intimas, conocimiento de } \\
\text { las fortalezas y debilidades propias. }\end{array}$ \\
\hline Musical & $\begin{array}{l}\text { Habilidad para producir y apreciar } \\
\text { ritmo, tono y timbre; apreciación de } \\
\text { las formas de expresión musical. }\end{array}$ \\
\hline Naturalista & $\begin{array}{l}\text { Habilidades de ordenación y } \\
\text { clasificación, reconocimiento de } \\
\text { secuencias de desarrollo, así como la } \\
\text { formulación de hipótesis, aplicadas } \\
\text { en forma práctica en el conocimiento } \\
\text { del medio. }\end{array}$ \\
\hline
\end{tabular}

\section{2) Procesamiento y Análisis de los de Datos}

Gráfico № 01: Histograma de frecuencias según nivel de desarrollo de inteligencias múltiples.

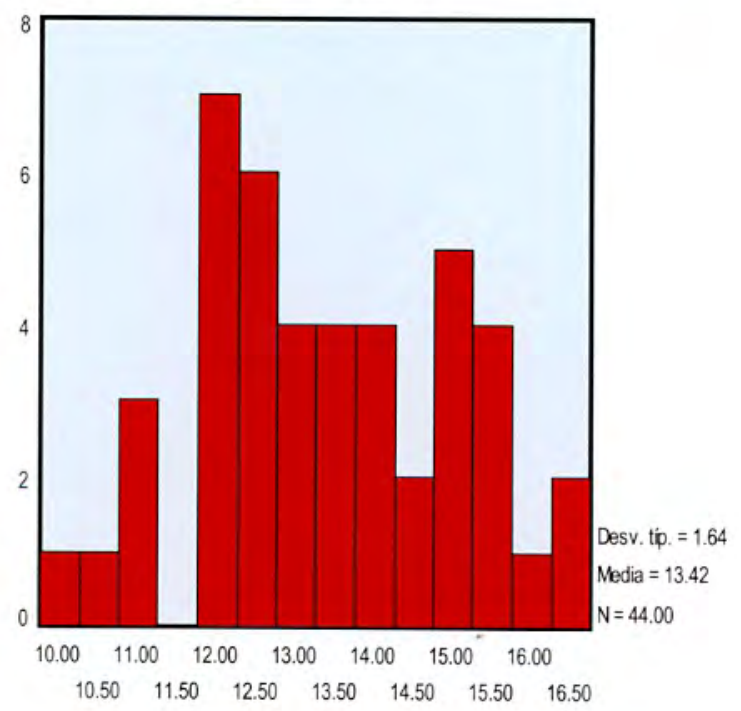

Inteligencias Múltiples
Interpretación: analizando el Gráfico 01 se puede deducir que:

sLa media del nivel desarrollo de las inteligencias múltiples de la muestra estudiada es de 13.42 .

*. Hay 23 alumnos que tienen puntuación por encima de la media (el $52 \%$ ). Hay 21 alumnos que tienen puntuación pordebajo de la media (el $48 \%$ ).

Gráfico $N^{\circ}$ 02: Histograma de frecuencias según Rendimiento Académico

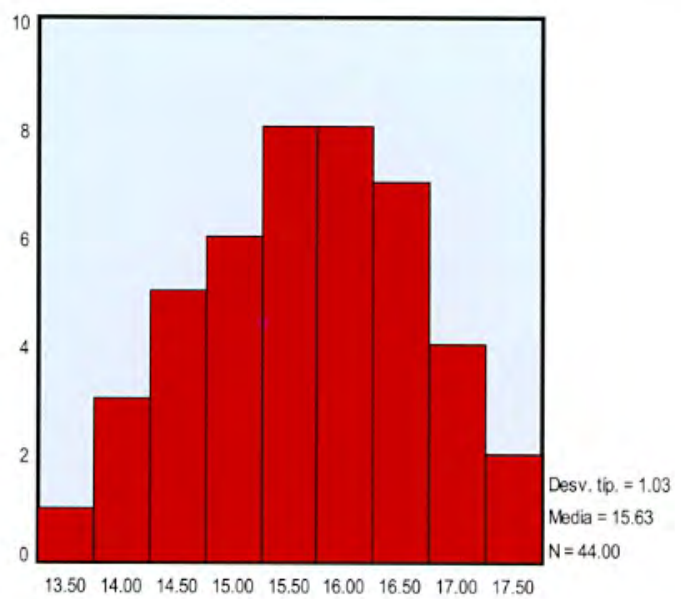

Rendimiento Académico

Interpretación: analizando el Gráfico 02 se puede deducir que:

La media del Rendimiento Académico de la muestra estudiada es de 15,63.

Hay 21 alumnos que tienen puntuación por encima de la media (el $48 \%$ ).

Hay 23 alumnos que tienen puntuación por debajo de la media (el $52 \%$ ).

\section{3) Contrastación de la Hipótesis General: $\mathrm{Hi}$}

Este análisis permitió probar que :

"Existe una correlación directa y significativa entre el nivel de desarrollo de las inteligencias múltiples y el Rendimiento Académico de los alumnos del primero y segundo grado de Secundaria de la Institución Educativa "Cristo Rey" de Tacna.

\section{Nuestro análisis utilizó el nivel de significación del 0,10.}

En nuestro caso el software estadístico SPSS nos presentó los siguientes resultados:

\section{Estadísticos descriptivos}

\begin{tabular}{|l|c|c|c|}
\hline & Media & $\begin{array}{c}\text { Desviación } \\
\text { típica }\end{array}$ & $\mathbf{N}^{\circ}$ \\
\hline Inteligencias Múltiples & 13,4188 & 1,63744 & 44 \\
\hline Rendimiento Académico & 15,6348 & 1,03193 & 44 \\
\hline
\end{tabular}




\section{4) Correlaciones}

\begin{tabular}{|l|l|c|c|}
\hline & & $\begin{array}{c}\text { Inteligencia } \\
\text { Múltiple }\end{array}$ & $\begin{array}{c}\text { Rendimiento } \\
\text { Académico }\end{array}$ \\
\hline Inteligencias Múltiples & Correlación de Pearson & 1 & .255 \\
& Sig. (bilateral) &. & .094 \\
& $\mathrm{~N}$ & 44 & 44 \\
Rendimiento Académico & Correlación de Pearson & .255 & 1 \\
& Sig. (bilateral) & .094 &. \\
& $\mathrm{~N}$ & 44 & 44 \\
\hline
\end{tabular}

Por lo tanto: se ACEPTA la hipótesis general $\mathrm{Hi}$ con un nivel de significancia de 0,10 .

Gráfico $N^{\circ}$ 03: Histograma de frecuencias según nivel de desarrollo de la inteligencia intrapersonal.

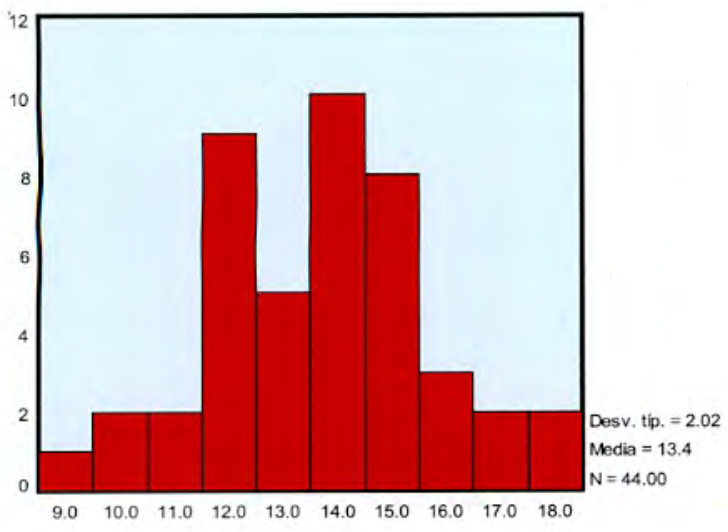

IM Intrapersonal

\section{Interpretación:}

La media del nivel de desarrollo de la inteligencia intrapersonal es de 13,4.

Hay 25 alumnos que tienen puntuación por encima de la media (el $57 \%$ ).

Hay 19 alumnos que tienen puntuación por debajo de la media (el $43 \%$ ).

Hay 19 alumnos que tienen puntuación por debajo de la media (el $43 \%$ ).

Gráfico No 04: Histograma de frecuencias según Rendimiento Académico en el Área de Educación Religiosa.

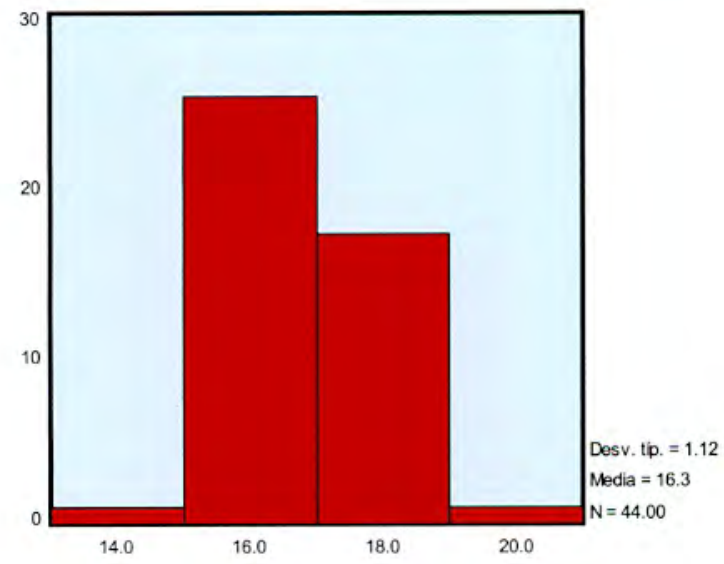

\section{Interpretación}

Hay 17 alumnos que tienen puntuación por encima de la media (el $39 \%$ )

Hay 27 alumnos que tienen puntuación por debajo de la media (el61\%)

\section{5) Contrastación de la Hipótesis Específica: H6}

Este análisis permitirá probar si "Existe una correlación directa y significativa entre el nivel de desarrollo de la inteligencia intrapersonal y el Rendimiento Académico en el curso de Educación Religiosa de los alumnos del primero y segundo grado de secundaria de la Institución Educativa "Cristo Rey" de Tacna. 0,05 .

Nuestro análisis ha utilizado el nivel de significación del

En nuestro caso el software estadístico SPSS nos presenta los siguientes resultados:

\section{Estadisticos Descriptivos}

\begin{tabular}{|l|c|c|c|}
\hline & Media & $\begin{array}{c}\text { Desviación } \\
\text { típica }\end{array}$ & $\mathbf{N}^{\circ}$ \\
\hline IM Intrapersonal & 13,4205 & 2.02296 & 44 \\
\hline RA Educación Religiosa & 16,2500 & 1.12322 & 44 \\
\hline
\end{tabular}

\section{Correlaciones}

\begin{tabular}{|l|l|r|r|}
\hline & & $\begin{array}{c}\text { Inteligencia } \\
\text { Múltiple }\end{array}$ & $\begin{array}{c}\text { Rendimiento } \\
\text { Académico }\end{array}$ \\
\hline IM Intrapersonal & Correlación de Pearson & 1 & $.326\left({ }^{*}\right)$ \\
\hline & Sig. (bilateral) &. & .031 \\
\hline & $\mathrm{N}$ & 44 & 44 \\
\hline RA Educación Religiosa & Correlación de Pearson & $.326\left(^{*}\right)$ & 1 \\
\hline & Sig. (bilateral) & .031 &. \\
\hline & $\mathrm{N}$ & 44 & 44 \\
\hline
\end{tabular}

* La correlación es significante al nivel 0,05 (bilateral).

Por lo tanto: se ACEPTAla hipótesis especifica H6.

\section{CONCLUSIONES}

1. El análisis de correlación de Pearson nos permite aceptar la hipótesis general $\mathrm{Hi}$ de la investigación con un nivel de significancia del 0,10, esto es, "existe una correlación directa y significativa entre el nivel de desarrollo de las inteligencias múltiples y el Rendimiento Académico de los alumnos del primero y segundo grado de la Institución Educativa "Cristo Rey" de Tacna".

2. El análisis de correlación de Pearson nos permite aceptar la hipótesis especifica $\mathrm{H} 6$ de la investigación con un nivel de significancia del 0,05, esto es, "existe una correlación directa y significativa entre el nivel de desarrollo de las inteligencias intrapersonal y el Rendimiento Académico en el curso de Educación Religiosa de los alumnos del primero y segundo grado de la Institución Educativa "Cristo Rey" de Tacna.

RA Educación Religiosa 


\section{RECOMENDACIONES}

Que investigaciones futuras relacionadas con la presente investigación puedan ser ampliadas y profundizadas y de Tipo Experimental, de tal manera que se obtengan resultados y propuestas orientadas a mejorar las condiciones de desarrollo personal de los estudiantes de la Institución Educativa "Cristo Rey "de Tacna.

Que los docentes se informen e indaguen sobre sus propias inteligencias múltiples y las de sus alumnos. Esto posibilitará una mejor selección de estrategias metodológicas.

Informar a los alumnos sobre sus inteligencias múltiples más predominantes en ellos, asi como sobre estrategias para mejorarlas, a fin de obtener mejores resultados en su aprendizaje.

Explicar a los padres de familia sobre las inteligencias múltiples más predominantes en sus hijos, afin de que les brinden el apoyo adecuado y obtener asi mejores resultados con ellos.

\section{BIBLIOGRAFÍA}

Armstrong, T. (2 001). Inteligencias Múltiples: Cómo descubrirlas $y$ estimularlas en sus hijos. ( $1^{\text {a }}$ Edic. en castellano). Bogotá: Norma.
Gardner, H. (1993). INTELIGENCIASMÚLTIPLES: Ia Teoria en la Práctica. (1 $1^{\mathrm{a}}$ Edic. en castellano). Barcelona: Paidos.

Gardner, H. (1 987). ESTUCTURAS DELAMENTE: $\left(1^{\circledR}\right.$ Edic. en castellano). México: Fondo de la Cultura Económica.

Navarro E. y Soto A. (2004). ¿Cómo estimular las inteligencias múltiples en el proceso de enseñanza y aprendizaje? Lima: Abedul.

Shneider, S. (2 004). Las Inteligencias Múltiples y el Desarrollo Personal. Colombia: Printer Colombiana S.A.

\section{Fuentes Hemerográficas}

Esquema de presentación del Trabajo de Investigación distribuido por la Institución Educativa "Cristo Rey" para el año 2007.

\section{Fuentes Electrónicas}

Guerrero, F. (s/f). Inteligencias Múltiples.

Recuperado de:

http://www.monografias.com/trabajos12/intmultintmult.sht $\mathrm{ml}$ [2007: 20 de Abrill]

Del Pino, J. (s/f). Influencia de las Inteligencias Múltiples en el rendimiento deportivo en futbol.

Recuperado de:

www.ugr.es/ setchift/docs/tesina_rendimientofutbol.doc[20 07: 20 de Abril] (Universidad de Granada) 